\title{
Formation of an Unusual Four-Membered Nitrogen Ring
}

Synthesis of

Materials and

Unnatural Products

\section{Key words}

diquinone-based cavitands

molecular machines

resorcin[4]arenes

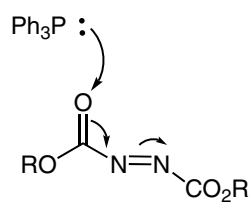

$\mathrm{R}=\mathrm{Et}, i-\mathrm{Pr}$

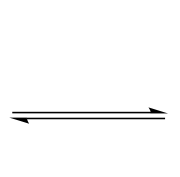<smiles>O=C([O-])[N-]N=C(O[Pb])O[Pb]</smiles>

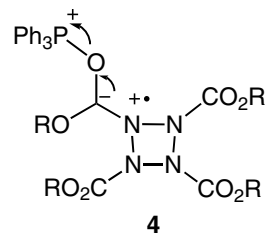

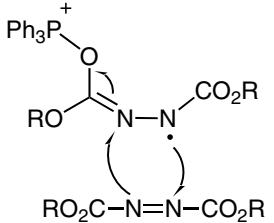

3<smiles>O=C(O)n1c(=O)n(C(=O)O)n1C(O)O[Pb]</smiles>

4
Significance: Above is one of three proposed mechanisms for the formation of the new radical cationic nitrogen four-membered ring $\mathbf{5}$. This tetrazetidine forms from allowing triphenylphosphine and diisopropyl azodicarboxylate to react. Previous studies had shown cursory evidence for the formation of a tetrazetidine by other methods, while this work offers more conclusive evidence in the form of EPR spectra and DFT calculations.
Comment: The reaction was monitored by EPR spectroscopy, which showed the presence and disappearance of $\mathbf{3}$ followed by the formation of $\mathbf{5}$. Thus, the authors claim that the above mechanism is the most plausible. The DFT calculations show electron density on the methine or methylene protons $(\mathrm{R}=\mathrm{Et}, i-\mathrm{Pr})$, which helps to rationalize hydrogen hyperfine coupling seen on the EPR spectra. 